\title{
An Empirical Study of Qualities of Association Rules from a Statistical View Point
}

\author{
Maryann Dorn*, Wen-Chi Hou*, Dunren Che*, and Zhewei Jiang*
}

\begin{abstract}
Minimum support and confidence have been used as criteria for generating association rules in all association rule mining algorithms. These criteria have their natural appeals, such as simplicity; few researchers have suspected the quality of generated rules. In this paper, we examine the rules from a more rigorous point of view by conducting statistical tests. Specifically, we use contingency tables and chi-square test to analyze the data. Experimental results show that one third of the association rules derived based on the support and confidence criteria are not significant, that is, the antecedent and consequent of the rules are not correlated. It indicates that minimum support and minimum confidence do not provide adequate discovery of meaningful associations. The chi-square test can be considered as an enhancement or an alternative solution.
\end{abstract}

Keywords: Data Mining, Association Rule Mining, Rule Evaluation, Chi-square Test

\section{Introduction}

Mining market basket data $[1,2,3,7]$ has received a great deal of attention in the recent past, partly due to its utility and partly due to the research challenges it presents. Market basket data typically consists of store items purchased on a per-transaction basis, but it may also consist of items bought by a customer over a period of time. The goal is to discover buying patterns, such as two or more items that are often bought together. Such finding could aid in business decision making. Association rules reflect a fundamental class of patterns that exist in the data. Consequently, mining association rules in market basket data has become an important problem in data mining.

In general, the task of mining all rules (or association rules) can be accomplished in two steps:

(1) Find all itemsets that are above a given minimum support ratio. The support ratio for an itemset is the percentage of the number of transactions that contain the itemset against the total number of transactions. Itemsets satisfying the minimum support (minsup) are called large (or frequent) itemsets, and all others small itemsets. This step is responsible for most of the computation time, and has been the focus of considerable work in developing fast algorithms [1, 2, 3, 6, 7, 16, 19, 21]. Agrawal \& Srikant [2, 3 ] have provided the initial foundation for this research problem.

(2) Use the large itemsets to generate the association rules. For example, both $\{\mathrm{A}, \mathrm{B}, \mathrm{C}, \mathrm{D}\}$ and $\{\mathrm{A}, \mathrm{B}\}$ are large itemsets. The association rule, $\mathrm{AB}=>\mathrm{CD}$, is derived if at least $\mathrm{c} \%$ of the transactions that contain $\mathrm{AB}$ also contain

\footnotetext{
Manuscript received July 6, 2007; revised December 8, 2007; accepted January 28, 2008.

Corresponding Author: Dunren Che

* Dept. of Computer Science, Southern Illinois University, Carbondale, Illinois, USA (\{mjdorn, hou, dche, zjiang $\} @$ cs.siu.edu)
}

$\mathrm{CD}$, where $\mathrm{c} \%$ is a pre-specified constant called minimum confidence (minconf).

There have been many algorithms developed to find association rules $[1,2,3,4,5,6,11,13,17,18,21]$. While association rule mining algorithms may be different in their efficiency, they all use minsup and minconf as the criteria to determine the validity of the rules. Due to their simplicity and natural appeals, few researchers have suspected the sufficiency of these criteria. In this research, we shall examine the rules derived based on these criteria in a more rigorous way by conducting statistical tests. The experimental results have shown that a surprising $30 \%$ of the rules satisfying the minsup and minconf are indeed insignificant statistically.

The rest of the paper is organized as follows. In Section 2 , we present some background on statistical testing. Section 3 discusses the experimental setup and the results. Section 4 is the conclusions.

\section{Background of Chi-Square Test for Independence}

Consider the market basket example from [6]. The focus is on the purchase of tea and coffee. Assume the minsup is $5 \%$ and minconf is $50 \%$. In Table 1 , rows tea and no tea corresponds to baskets (or transactions) that do and do not, respectively, contain tea; and similarly columns coffee and no_coffee corresponds to baskets that do and do not contain coffee. The numbers in the table represent percentage of baskets. A potential association rule 'tea $=>$ coffee' reads: "When people buy tea, they are also likely to buy coffee". The support for this rule is $20 \%$, which is fairly high. The confidence, defined by the conditional probability that a customer buys coffee, given that he/she also buys tea, i.e., $\mathrm{p}$ [tea and coffee] / $\mathrm{p}$ [tea], is $20 / 25=0.8$ or $80 \%$, which is also high. Since the support and confidence satisfy the 
pre-specified minsup (5\%) and minconf $(50 \%)$, the rule is accepted as valid in the support-confidence framework.

Table 1. A 2 by 2 Contingency Table.

\begin{tabular}{|l|c|c|c|}
\hline & coffee & no_coffee & $\sum$ row \\
\hline tea & 20 & 5 & 25 \\
\hline no_tea & 70 & 5 & 75 \\
\hline$\sum$ col & 90 & 10 & 100 \\
\hline
\end{tabular}

The chi-square $\left(\chi^{2}\right)$ test is a non-parametric statistical method that can be used to test independence among attributes. It is reliable under a fairly permissive set of assumptions. This approximation breaks down when the expected values are small. As a rule of thumb, statistics texts $[9,15]$ recommend the use of chi-square test only if (1) all cells in the contingency table have expected values greater than 1, and (2) at least $80 \%$ of the cells in the contingency table have expected values greater than 5 . With small expected cell values, the usual alternative is to combine or collapse cells $[9,10,15]$.

The $\chi^{2}$ statistics is approximately distributed with a chisquare distribution, which is often tabulated in statistics texts. The $\chi^{2}$ value is computed from a contingency table, which records the counts or frequencies of different combinations of attribute values. The $\chi^{2}$ statistics has a parameter called degrees of freedom associated with it. In Table 1, we show a 2 by 2 contingency table.

Given an $r \times c$ contingency table, the $\chi^{2}$ is computed as follows:

$$
\chi^{2}=\sum \frac{\left(O_{i j}-E_{i j}\right)^{2}}{E_{i j}}
$$

where $\sum$ is taken over all cells of the table; $O_{j j}$ is the observed count for cell $i j$, and $E_{i j}$ is the expected count for cell $i j$, which is calculated as $E_{i j}=n_{i} n_{j} / n$, where $n_{i}, n_{j}$ are the total counts of row $i$ and column $j$, respectively, and $n$ is the total count of all the cells. The table has a (r$1) \times(c-1)$ degree of freedom.

When Table 1 is tested by chi-square statistics, the result, $\chi^{2}=3.703703$ with degree of freedom $=1$, is nonsignificant at $95 \%$ confidence level; it indicates that tea and coffee are independent variables. In other words, tea is not a determining factor whether people will buy coffee or not. Obviously, there is some discrepancy between the conclusions drawn from the two methods.

Potential advantages of the $\chi^{2}$ statistics over the commonly used support-confidence framework [6] are:

(1) The use of the chi-square significance test for independence is more solidly founded in statistical theory. In particular, there is no need to choose adhoc values of support and confidence.

(2) The chi-square statistic simultaneously and uniformly takes into account all possible combinations of the presence and absence of the various attributes being examined as a group.

(3) The chi-square test at a given significance level is upward closed. In other words, if an i-itemset is correlated, all its supersets are also correlated.

\section{Experiments}

In this section, we present the experimental design and analyze its results.

\subsection{Experimental Design}

Four synthetic data sets are generated using the IBM/Quest data generator [3], which has been widely used for evaluating association rule mining algorithms. The data sets generated are then fed into CBA/DBII data mining system [12] to generate association rules. Finally, contingency tables are constructed using Microsoft Excel, and the chi-square statistical tests are conducted on the association rules generated.

The synthetic transactions generated are supposed to mimic the transactions in the retail environment. The model of the "real" world is that people tend to buy sets of items together. A transaction may contain more than one large itemset. For example, a customer might place an order for a dress and jacket when ordering sheets and pillowcases, in which the dress and jacket form one large itemset and sheets and pillowcases together form another large itemset. Transaction sizes are typically clustered around a mean with a few transactions having many items. Typical sizes of large itemsets are also clustered around a mean, with a few itemsets having a large number of items.

Some of the options of the data generator are as follows:

-ntrans

-tlen Average items per transaction (default: 10)

-nitems

-npats number of patterns (default: 10000)

-patlen average length of maximal pattern $($ default $=4)$

-corr correlation between patterns (default 0.25 )

-conf average confidence in a rule $($ default $=0.75)$

The transaction size is picked from a Poisson distribution [3] with mean $\mu$ equal to -tlen. Note that if each item is chosen with the same probability $\mathrm{P}$ and there are $\mathrm{N}$ items, the expected number of items in a transaction is given by a binomial distribution with parameters $\mathrm{N}$ and $\mathrm{P}$, and is approximated by a Poisson distribution with mean NP.

In order to model the phenomenon that large itemsets often have common items, some fraction of the items in the subsequent itemsets are chosen from the previous itemset generated. It uses an exponentially distributed random variable [3] with mean equal to the correlation level (-corr) to decide this fraction for each itemset. The remaining items are picked at random. In the datasets applied in this study, the correlation level was set to 0.25 . Agrawal \& Srikant [3] ran some experiments with the correlation level set to 0.5 and 0.75 but did not find much difference in the nature of the performance results.

Each itemset in the database has a weight associated with it, which corresponds to the probability that this 
itemset will be picked. This weight is picked from an exponential distribution with unit mean, and is then normalized so that the sum of the weights for all the itemsets is 1 .

Four synthetic datasets are generated with slightly different options for comparison, such as, number of transactions, number of items, and average confidence for rules (see Table 2). The other common factors are 'average transaction length (5)', 'number of patterns (100)', 'average length of pattern (4)', and 'correlation between consecutive patterns $(0.25)$ '.

Table 2: Four Datasets

\begin{tabular}{|c|c|c|c|}
\hline $\begin{array}{c}\text { Data } \\
\text { sets }\end{array}$ & $\begin{array}{c}\text { Num of } \\
\text { transactions }\end{array}$ & Num of items & $\begin{array}{c}\text { Avg. Conf. } \\
\text { level }\end{array}$ \\
\hline 1 & 2217 & 20 & 0.5 \\
\hline 2 & 5188 & 20 & 0.5 \\
\hline 3 & 5029 & 40 & 0.5 \\
\hline 4 & 5096 & 40 & 0.75 \\
\hline
\end{tabular}

Once the data sets are generated, the CBA system [12, $14]$ is used to generate association rules that satisfy the given minsup and minconf.

\subsection{Experimental Results and Chi-Square Test}

First, we discuss the effects of minsup and minconf on the numbers of large itemsets and rules produced. Then, we will examine the quality of the rules using the chi-square test.

\subsubsection{Effects of Minsup and Minconf}

The value of minimum support (minsup) controlls the number of large itemsets. Table 3 shows the drastic reduction of the number of large itemsets when minsup increases from $1 \%$ to $5 \%$. For example, in the 2217 transaction dataset, the number of large itemsets drops from 3401 to 408 .

The size of dataset can also affect the level of minsup used significantly. The larger the data size, the smaller the minsup is needed to provide a controllable size of large itemsets. For example, the datasets with over 5000 transactions generate fewer large itemsets than the dataset with 2217 transactions for the same minsup (see Table 3).

Table 3. Number of Large Itemsets at Various Minsup Levels

\begin{tabular}{|cc|c|c|c|c|c|c|}
\hline \multicolumn{2}{|c|}{ Data set } & $1 \%$ & $2 \%$ & $5 \%$ & $10 \%$ & $15 \%$ & $20 \%$ \\
\hline 1 & $(2217)$ & 3401 & 1488 & 408 & 131 & 65 & 36 \\
\hline 2 & $(5188)$ & 1836 & 808 & 246 & 84 & 37 & 24 \\
\hline 3 & $(5029)$ & 1513 & 521 & 118 & 40 & 17 & 11 \\
\hline 4 & $(5096)$ & 1600 & 531 & 118 & 40 & 17 & 11 \\
\hline
\end{tabular}

The relationship among minsup, minconf, and the number of the association rules is shown in Tables 4, 5, 6, and 7 for each dataset respectively. The confidence level also affects the number of association rules generated significantly. The higher the level of minconf becomes, the smaller the number of association rules sustains. The determination of minconf cutoff point is a major problem. When the data size is large, a lower minsup is preferred. If the minsup is set too high, combined with a minconf at $75 \%$ or $90 \%$, often it generates no association rule. Table 5 shows that at minsup $=1 \%$ and minconf $=50 \%$, it only generates 1487 rules, and with minconf $=75 \%$ it generates 217 rules. But when the minsup moves up to $2 \%$, with minconf $=75 \%$, it only generates 43 rules.

Table 4. Number of Transactions: 2217, Average Confidence: 0.5

\begin{tabular}{|c|c|c|c|c|c|c|c|c|c|c|c|c|}
\hline minsup & \multicolumn{3}{|c|}{$1 \%$} & \multicolumn{3}{|c|}{$5 \%$} & \multicolumn{3}{c|}{$10 \%$} & \multicolumn{3}{c|}{$15 \%$} \\
\hline minconf & $50 \%$ & $75 \%$ & $90 \%$ & $50 \%$ & $75 \%$ & $90 \%$ & $50 \%$ & $75 \%$ & $90 \%$ & $50 \%$ & $75 \%$ & $90 \%$ \\
\hline Itemsets & 3401 & 3401 & 3401 & 408 & 408 & 408 & 131 & 131 & 131 & 65 & 65 & 65 \\
\hline Rules & 3169 & 1261 & 89 & 358 & 38 & 0 & 112 & 3 & 0 & 50 & 0 & 0 \\
\hline
\end{tabular}

Table 5. Number of Transactions: 5188, Average Confidence: 0.5

\begin{tabular}{|c|c|c|c|c|c|c|c|c|c|c|c|c|}
\hline minsup & \multicolumn{3}{|c|}{$1 \%$} & \multicolumn{3}{|c|}{$5 \%$} & \multicolumn{3}{|c|}{$10 \%$} & \multicolumn{3}{c|}{$15 \%$} \\
\hline minconf & $50 \%$ & $75 \%$ & $90 \%$ & $50 \%$ & $75 \%$ & $90 \%$ & $50 \%$ & $75 \%$ & $90 \%$ & $50 \%$ & $75 \%$ & $90 \%$ \\
\hline Itemsets & 1836 & 1836 & 1836 & 246 & 246 & 246 & 84 & 84 & 84 & 37 & 37 & 37 \\
\hline Rules & 1487 & 217 & 9 & 176 & 5 & 0 & 57 & 0 & 0 & 19 & 0 & 0 \\
\hline
\end{tabular}

Table 6. Number of Transactions: 5029, Average Confidence: 0.5

\begin{tabular}{|c|c|c|c|c|c|c|c|c|c|c|c|c|}
\hline minsup & \multicolumn{3}{|c|}{$1 \%$} & \multicolumn{3}{c|}{$5 \%$} & \multicolumn{3}{c|}{$10 \%$} & \multicolumn{3}{c|}{$15 \%$} \\
\hline minconf & $50 \%$ & $75 \%$ & $90 \%$ & $50 \%$ & $75 \%$ & $90 \%$ & $50 \%$ & $75 \%$ & $90 \%$ & $50 \%$ & $75 \%$ & $90 \%$ \\
\hline Itemsets & 1513 & 1513 & 1513 & 118 & 118 & 118 & 40 & 40 & 40 & 17 & 17 & 17 \\
\hline Rules & 576 & 247 & 30 & 21 & 0 & 0 & 6 & 0 & 0 & 3 & 0 & 0 \\
\hline
\end{tabular}

Table 7. Number of Transactions: 5096, Average Confidence: 0.75

\begin{tabular}{|c|c|c|c|c|c|c|c|c|c|c|c|c|}
\hline minsup & \multicolumn{3}{|c|}{$1 \%$} & \multicolumn{3}{|c|}{$5 \%$} & \multicolumn{3}{|c|}{$10 \%$} & \multicolumn{3}{c|}{$15 \%$} \\
\hline minconf & $50 \%$ & $75 \%$ & $90 \%$ & $50 \%$ & $75 \%$ & $90 \%$ & $50 \%$ & $75 \%$ & $90 \%$ & $50 \%$ & $75 \%$ & $90 \%$ \\
\hline Itemsets & 1600 & 1600 & 1600 & 118 & 118 & 118 & 40 & 40 & 40 & 17 & 17 & 17 \\
\hline Rules & 677 & 204 & 64 & 24 & 0 & 0 & 8 & 0 & 0 & 3 & 0 & 0 \\
\hline
\end{tabular}




\subsubsection{Chi-Square Tests for Independence}

The results from the chi-square tests for independence constitute the major findings of this study. Note that all chisquare tests conducted in this study adopted the commonly used significance level, $\mathrm{p} \leq 0.05$, as a cutoff point. The critical value is 3.841 for 1 degree of freedom.

In the following, we use the dataset with 2217 transactions as an example. Each transaction has three attributes: Transaction number, Customer ID, and item. To facilitate interpretation, 20 items are randomly assigned different labels.

The first experiment was conducted under minsup $=10 \%$ and $\operatorname{minconf}=50 \%$. CBA produced 131 large itemsets and 112 association rules as shown in Table 4 under the $10 \%$ (minsup) and 50\% (minconf) column. All 112 rules generated were then tallied into 2 by 2 contingency tables and tested by the chi-square test for independence. The results show that 37 out of 112 rules are not significant, in other words, 37 rules show that there is no relationship between their antecedents and the consequents.

With the same dataset, when the minsup was reduced to $5 \%$ and the minconf was kept the same level at $50 \%$, that is, the restriction was loosened, the CBA program produced 408 large itemsets and 385 association rules as shown in Table 4 in the $5 \%$ and $50 \%$ column. Out of these 385 rules, 134 rules are not significant. The previous 37 rules are part of these 134 rules. It again shows that no relation between the antecedents and consequents in one third of the association rules derived by the support-confidence framework. The results demonstrate a striking discrepancy between the support-confidence framework and the chisquare statistical tests.

\subsection{Analysis}

We use examples to see why some of the rules generated according to minsup and minconf may not be valid statistically.

\subsubsection{Sufficiency of Minsup and Minconf}

The following two examples illustrate one interesting phenomenon. The support and the confidence ratios for Rule 175 (Example 1) and 169 (Example 2) are exactly the same. With the same cut-off minsup and minconf, the two rules are both valid. However, they have the opposite fate in statistical tests. The $\chi^{2}$ value for Rule 175 , as shown in Table 8 , is not significant $(\mathrm{p}>0.05)$. It indicates that 'buying apple and ketchup' is independent from 'buying

Example 1. Non-Significant Relationship

Table 8. Chi-square Test vs. Minsup/Minconf

\begin{tabular}{|c|c|c|c|c|c|}
\hline \multicolumn{7}{|c|}{ Rule 175: AP/KP $=>$ PA } \\
\hline & R & NO_R & T_ROW & SUP_L & CONF \\
\hline L & 111 & 74 & 185 & $8.34 \%$ & $60.00 \%$ \\
\hline NO_L & 1134 & 898 & 2032 & & \\
\hline T_COL & 1245 & 972 & 2217 & & \\
\hline SUP_R & $56.16 \%$ & \multicolumn{7}{|c|}{$\mathrm{p} \leq 0.271182286$} & $5.01 \%$ & \\
\hline \multicolumn{7}{|c|}{} \\
\hline
\end{tabular}

Example 2. Significant Relationship

Table 9. Chi-square Test vs. Minsup/Minconf

\begin{tabular}{|c|c|c|c|c|c|}
\hline \multicolumn{7}{|c|}{ Rule 169: AP/KP $=>$ OV } \\
\hline & R & NO_R & T_ROW & SUP_L & CONF \\
\hline L & 111 & 74 & 185 & $8.34 \%$ & $60.00 \%$ \\
\hline NO_L & 1037 & 995 & 2032 & & \\
\hline T_COL & 1148 & 1069 & 2217 & & \\
\hline SUP_R & $51.78 \%$ & & & $5.01 \%$ & \\
\hline \multicolumn{7}{|c|}{$p \leq 0.019456897$} \\
\hline
\end{tabular}

pamper'. In Example 2, the $\chi^{2}$ value for Rule 169 is significant $(\mathrm{p}<0.05)$. It indicates that there is a relationship between 'buying an oven' and 'buying apple and ketchup'. It implies that merely depending on minsup and minconf as a constraint factor may not provide a reliable basis for judging the association.

\subsubsection{Uni-Directional vs. Bi-Directional}

The association rule in the Apriori algorithm is unidirectional. A rule $\mathrm{X}=>\mathrm{Y}$ does not necessarily imply the rule $\mathrm{Y}=>\mathrm{X}$. In addition, it cannot detect negative implication, such as, buying product $X$ and not buying product Y. However, the chi-square statistic simultaneously and uniformly takes into account all possible combinations of the presence and absence of the various attributes. We shall illustrate these in the following examples.

Example 3. Bi-directional Relationship

Table 10. Chi-square Test on Bi-directional Relationship

\begin{tabular}{|c|c|c|c|c|c|}
\hline \multicolumn{7}{|c|}{ Rule 1: $\mathrm{T}=>$ S } \\
\hline & S & NO_S & T_ROW & SUP & CONF \\
\hline T & 36 & 19 & 55 & $29.10 \%$ & $65.45 \%$ \\
\hline NO_T & 112 & 22 & 134 & & \\
\hline T_COL & 148 & 41 & 189 & & \\
\hline & $78.31 \%$ & & & $19.05 \%$ & \\
\hline & \multicolumn{7}{|c}{ P $\leq 0.01$} \\
\hline
\end{tabular}

(a). Rule: $\mathrm{T}=>\mathrm{S}$

\begin{tabular}{|c|c|c|c|c|c|}
\hline \multicolumn{7}{|c|}{ Rule 2: $\quad$ S $=>$ T } \\
\hline & T & NO_T & T_ROW & SUP & CONF \\
\hline S & 36 & 112 & 148 & 78.31 & $24.32 \%$ \\
\hline NO_S & 19 & 134 & 41 & & \\
\hline T_COL & 55 & 134 & 189 & & \\
\hline \multicolumn{7}{|c|}{} & $29.10 \%$ & & & 19.05 & \\
\hline$\chi^{2}=7.54330480 .01$ \\
\hline
\end{tabular}

(b). Rule: $\mathrm{S}=>\mathrm{T}$

Example 3 shows that at minconf $=50 \%$, the confidence of Rule 1: $\mathrm{T}=>\mathrm{S}$ is high $(65.45 \%)$, so Rule 1 is accepted as an association rule. But the confidence of Rule $2: \mathrm{S}=>\mathrm{T}$ is too low $(24.32 \%)$, so Rule 2 is pruned off. However, the $\chi^{2}$ values of both Rule 1 and Rule 2 are the same and their $\mathrm{P}$ values are all less than 0.05 , which implies that $\mathrm{T}$ and $\mathrm{S}$ are correlated and $\mathrm{S}=>\mathrm{T}$ should have been accepted as a rule.

It is also useful that the chi-square test can detect negative implication. Example 4 illustrates this case. The $\chi^{2}$ values for both $\mathrm{L}=>\mathrm{R}$ and $\mathrm{L}=>\left(\mathrm{NO} \_\mathrm{R}\right)$ are the same. Since $\mathrm{p}>0.05$, it indicates that $\mathrm{L}$ and $\mathrm{R}$ are two independent 
Example 4. Negative Relationship.

Table 11. Chi-square Test on Negative Relationship

\begin{tabular}{|c|c|c|c|c|c|}
\hline \multicolumn{7}{|c|}{ Rule 3: L $=>$ R } \\
\hline & R & NO_R & T_ROW & SUP & CONF \\
\hline L & 29 & 26 & 55 & $29.10 \%$ & $52.73 \%$ \\
\hline NO_L & 77 & 57 & 134 & & \\
\hline T_COL & 106 & 83 & 189 & & \\
\hline \multicolumn{7}{|c|}{$\mathrm{p} \leq 0.55128125$} & $15.34 \%$ \\
\hline & $56.08 \%$ & \multicolumn{7}{|c|}{} \\
\hline
\end{tabular}

\begin{tabular}{|c|c|c|}
\hline \multicolumn{3}{|c|}{ RULE 3: (Expected) } \\
\hline & $\mathrm{R}$ & NO R \\
\hline $\mathrm{L}$ & 30.85 & 24.15 \\
\hline NO_L & 75.15 & 58.85 \\
\hline & & \\
\hline \multicolumn{3}{|c|}{$\mathrm{p} \leq 0.55128125$} \\
\hline
\end{tabular}

(a) Rule 3: $\mathrm{L}=>\mathrm{R}$

\begin{tabular}{|c|c|c|c|c|c|}
\hline \multicolumn{7}{|c|}{ Rule 4: L => NO_R } \\
\hline & NO_R & R & T_ROW & SUP & CONF \\
\hline L & 26 & 29 & 55 & $29.10 \%$ & $47.27 \%$ \\
\hline NO_L & 57 & 77 & 134 & & \\
\hline T_COL & 83 & 106 & 189 & & \\
\hline \multicolumn{7}{|c|}{$\mathrm{p} \leq 0.55128125$} & $13.76 \%$ \\
\hline & $43.92 \%$ & & & & \\
\hline
\end{tabular}

\begin{tabular}{|c|c|c|}
\hline \multicolumn{3}{|c|}{ RULE 4: (Expected) } \\
\hline & NO_R & R \\
\hline L & 24.15 & 30.85 \\
\hline NO_L & 58.85 & 75.15 \\
\hline & & \\
\hline \multicolumn{3}{|c|}{ p $\leq 0.55128125$} \\
\hline
\end{tabular}

(b) Rule 4: $\mathrm{L}=>\mathrm{NO}$ -

attributes. However, at minconf $=50 \%$, the confidence of the Rule 3: $\mathrm{L} \Rightarrow \mathrm{R}$ is high $(52.73 \%)$, so it is accepted as an association rule; but the confidence of Rule 4: $\mathrm{L}=>\mathrm{NO} R$ is low (47.27\%), so Rule 4 is pruned off.

\subsection{Enhancing the Support-confidence Framework}

The support-confidence framework can be enhanced by incorporating the chi-squared test. In fact, the required statistics for conducting chi-squared tests can be obtained with little effort as follows. Let $|\mathrm{T}|$ be the total number of transactions in the database. Let $\mathrm{X}$ be an itemset and $\left|\mathrm{T}_{\mathrm{X}}\right|$ (or the support of $\mathrm{X}$ ) the number of transactions containing $\mathrm{X}$. Let $\mathrm{X} \cup \mathrm{Y}$ be an itemset that contains all the items in $\mathrm{X}$ or $\mathrm{Y}$. Assume $\mathrm{X}, \mathrm{Y}$, and $\mathrm{X} \cup \mathrm{Y}$ are identified as frequent itemsets in the process of deriving frequent itemsets. Then, the required statistics for $\mathrm{X}$ but_not_Y can be computed, based on the set theory, as $\left|\bar{T}_{X}\right|-\left|T_{X} \cup_{Y}\right|$, Y_but_not_X as $\left|\mathrm{T}_{\mathrm{Y}}\right|-\left|\mathrm{T}_{\mathrm{X}} \cup \mathrm{Y}\right|$, and not_X_and_not_Y as $|\mathrm{T}|-\left|\mathrm{T}_{\mathrm{X}}\right|-$ $\left|\mathrm{T}_{\mathrm{Y}}\right|+\left|\mathrm{T}_{\mathrm{X}} \cup \mathrm{Y}\right|$. Therefore, given a minimum support requirement, all rules derived by the support-confidence framework can be tested using the chi-square statistics on the fly without much effort. With the incorporation of the chi-squared tests, the derived rules should be more reliable statistically.

\section{Conclusions}

We present in this paper an empirical study on the validity of association rules derived based on the minsup and minconf criteria. The results of this study strongly suggest that minsup and minconf do not provide adequate discovery of associations. From the results of chi-square tests, we conclude as follows.

(1) Minsup and minconf can significantly cut down the number of rules generated but does not necessarily only cut off those insignificant rules.

(2) Association rules derived are not very reliable in making inference such as "people who buy product A tend to buy product B." Approximately, one-third of such association rules, discovered with a minconf at $50 \%$ and minsup at either $5 \%$ or $10 \%$, fail to show that there exists statistical relationship between the antecedents and the consequents.

(3) The relationship between minsup and minconf was not decisive. High level of minconf $(70 \%$ above) in general implies significant relationship with either high or low minsup. Mid range of minconf mixed with either mid or high level of minsup could produce either significant or not-significant outcomes.

The chi-squared test has demonstrated itself as a more prudent way to discover correlations. The test can also be easily incorporated into the support-confidence framework (as discussed in Section 3.4). Combining the efficient algorithms for deriving frequent itemsets of the supportconfidence framework with the theoretically sound chisquared test, building an efficient and reliable data mining system is achievable.

\section{References}

[1] Agrawal, R., Imielinski, T., and Swami, A. "Mining Association Rules Between Sets of Items in Large Databases," In Proc. of the ACM-SIGMOD Conf. on Management of Data, Washington, D. C., 1993, pp. 207-216.

[2] Agrawal R., Srikant, R. "Fast algorithms for Mining Association Rules," In Proc. of the $20^{\text {th }}$ VLDB 
Conference, Santiago, Chile, 1994, pp. 487-499.

[3] Agrawal, R. and Srikant, R. "Fast Algorithms for Mining Association Rules," IBM Research Report RJ9839, June 1994. IBM Almaden Research Center, San Jose, CA.

[4] Bayardo, R. J. and Agrawal, R. "Mining the Most Interesting Rules," In Proc. of the Fifth ACM SIGKDD Conf. on Knowledge Discovery and Data Mining, 1999, pp.145-154.

[5] Bayardo, R., Agrawal, R, and Gunopulos, D. "Constraint-Based Rule Mining in Large, Dense Databases," In Proc. of the 15th Int'l Conf. on Data Engineering, 188-197, 1999.

[6] Brin, S. Motwani, R. and Silverstein, R. "Beyond Market Basket: Generalizing Association Rules to Correlations." SIGMOD-97, 1997, 265-276.

[7] Brin, S., Motwani, R., Ullman, J., and Tsur, S. "Dynamic Itemset Counting and Implication Rules for Market Basket Data." In Proc. of the 1997 ACM-SIGMOD Int'l Conf. on the Management of Data, 1997, 255-264.

[8] Ganti, V., Gebrke, and Ramakrishnan, R. "Mining Very Large Databases," Computer, Vol. 32, No. 8, Aug. 1999, pp. 38-45.

[9] Glass, G. V. and Hopkins, K. D. Statistical Methods in Education and Psychology. ( $2^{\text {nd }}$ ed. $)$ Prentice Hall, New Jersey, 1984.

[10] Gokhale, D. V. and Kullback, S. The Information in Contingency Tables. Marcel Dekker Inc., New York, 1978.

[11] Han, J. and Fu, Y. "Discovery of multiple-level association rules from large databases." VLDB-95.

[12] Liu B., Hsu W., and Ma Y. "Pruning and Summarizing the Discovered Associations, " in Proc. of the ACM SIGKDD Int'l Conference on Knowledge Discovery \& Data Mining, San Diego, CA, 1999.

[13] Liu B., Hsu W., and Ma Y. "Mining Association Rules with Multiple Minimum Supports" in Proc. of the ACM SIGKDD Int'l Conference on Knowledge Discovery \& Data Mining, 1999.

[14] Liu B., Hsu W., Wang K., and Chen S. "Mining Interesting Knowledge Using DM-II" in Proc. of the ACM SIGKDD Int'l Conference on Knowledge Discovery \& Data Mining, 1999.

[15] Mason, R. D., Lind, D. A., and Marchal, W. G. STATISTICS: An Introduction, $5^{\text {th }}$ ed. Duxbury Press, 1998.

[16] Park, J. S.; Chen, M.-S.; and Yu, P. S. An Effective Hash Based Algorithm for Mining Association Rules. In Proc. of SIGMOD Conf. on the Management of Data, 1995, pp 175-186.

[17] Srikant, R. and Agrawal, R. "Mining Generalized Association Rules," In Proc. of the 21st Int'l Conf. on VLDB, 1995, pp. 407-419.
[18] Srikant, R. and Agrawal, R. Mining Generalized Association Rules. IBM Research Report RJ9963, June 1995. IBM Almaden Research Center, San Jose, CA.

[19] Srikant, R., Vu, Q., and Agrawal, R. "Mining Association Rules with Item Constraints," In Proc. of the Third Int'l Conf. on Knowledge Discovery in Databases and Data Mining, 1997, pp. 67-73.

[20] Toivonen H. "Sampling Large Databases for Association Rules," In Proc. of the $22^{\text {th }}$ VLDB Conference, Mumbai, India, 1996, pp. 134-144.

[21] Zaki, M. J.; Parthasarathy, S.; Ogihara, M.; and Li, W. New Algorithms for Fast Discovery of Association Rules. In Proc. of the Third Int'l Conf. on Knowledge Discovery in Databases and Data Mining, 1997, pp. 283-286.

\section{Maryann Dorn}

She received a M.S degree in Computer Science from Southern Illinois University in 2000.

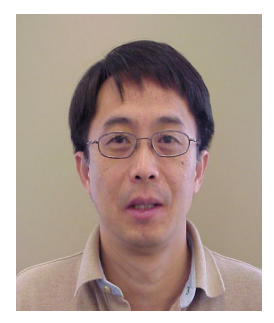

\section{Wen-Chi Hou}

He received a Ph.D. degree in Computer Sci. \& Eng. from Case Western Reserve Univ., Cleveland Ohio, in 1989. He is an associated professor at Southern Illinois University Carbondale, USA. His interests are in database $\&$ data mining.

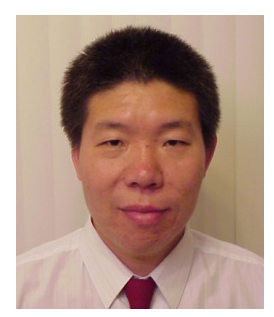

\section{Dunren Che}

He received his Ph.D. in Computer Science from BUAA China in 1994. He is an assistant Professor at Southern Illinois University Carbondale, U.S.A. His main interests are in database and data mining.

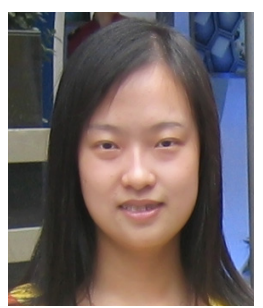

\section{Zhewei Jiang}

She is currently a Ph.D. student in the Department of Computer Science at Southern Illinois University, Carbondale Illinois, USA. Her research interests include database and data mining. 